\section{DISCRETE-TO-BETA: A FORTRAN IV program for converting discrete prior distributions into beta prior distributions}

\author{
JAMES W. GENTRY \\ College of Business Administration \\ Oklahoma State University, Stillwater, Oklahoma 74074
}

The beta distribution is frequently used when one wants to make Bayesian revisions about priors, as is discussed in Till (1977). The use of continuous rather than discrete priors makes the decision framework less artificial and computationally more efficient. However, unfamiliarity with the beta distribution causes added assessment problems (Corless, 1972; Gentry, Bonczkowski, \& Caldwell, in press). Consequently, training in the use of the beta distributions is needed before using the distribution in a decision-making framework. One means of obtaining the needed familiarity with the beta distribution is to provide information on corresponding discrete and beta distributions.

The Program. DISCRETE-TO-BETA is a FORTRAN IV program that finds a beta distribution corresponding to the input discrete distribution through the method of moments. The two distributions are plotted so that the user can get a better idea of their correspondence.

Input. The program requires the input of the discrete states of the world in decimal form (the hypothesized proportions) and the prior probability that each state is the true one.
Output. The program generates the mean and variance of the discrete distribution and the values of the estimates of the beta parameters that provide the same mean and variance values for the beta distribution. The program also plots the distributions.

Restriction. Program DISCRETE-TO-BETA is written in FORTRAN IV, so any computer system that supports FORTRAN IV can use it. It takes $1.28 \mathrm{sec}$ to run on the IBM 370-158 computer.

Availability. A copy of the listing, documentation, and sample of the output can be obtained free of charge by writing to Jim Gentry, College of Business Administration, Oklahoma State University, Stillwater, Oklahoma 74074 .

\section{REFERENCES}

Conless, J. C. Assessing prior distributions for applying Bayesian statistics in auditing. Accounting Review, 1972, 47, $556-566$.

Gentry, J. W., Bonczkowski, M. H., \& Caldwell, C. W. The use of program BAYAUD in the teaching of audit sampling. Journal of Exponential Learning and Simulation, in press.

TILL, A. BETA: A FORTRAN IV program for calculating the mean, standard deviation, and the mode of the prior and posterior beta distributions. Behavior Research Methods \& Instrumentation, 1977, 9, 543-544.

(Accepted for publication October 30, 1978.)

\title{
ERRATUM
}

Aaronson, D., and Grupsmith, E. The SIMPLE T-Scope. Behavior Research Methods \& Instrumentation, 1978, $10(6), 761-763$. Two paragraphs were omitted from the article. The conclusion is as follows:

\section{COPYING SIMPLE}

The bulk of our SIMPLE hardware was purchased about 10 years ago. If someone wanted to set up a roughly equivalent system today, it would probably be based on a PDP-8/e or a PDP-11/03. Floppy disks would be substituted for our paper tape and/or DECtape systems. A package including Teletype (or DECwriter), interface, digital $\mathrm{I} / 0$, clocks, relays, sense switches, and $16 \mathrm{~K}$ words of memory would cost $\$ 10,000$ to $\$ 15,000$, depending on the details. The visual display terminal would add another $\$ 2,000$ to $\$ 4,000$. During the next 2 years, we will be modifying SIMPLE to be used on a PDP.11/34.

The logical structure of the SIMPLE operating system and of the individual user commands is easily transportable to other machines. However, the detailed assembly language coding of some individual commands is highly hardware dependent. For example, subroutines that deal with the visual displays are dependent on the particular display logic as well as its timing, line length, and page size. People who want to inquire about setting up a SIMPLE system at their own laboratory can contact either of the authors. Copies of our current paper tape or DEC tape SIMPLE operating systems can be provided with the understanding that most users would have to reprogram the hardware-dependent functions. However, interested researchers should first read the three references mentioned in the first paragraph of this paper for a rather full description of SIMPLE. 